\title{
Fundamentals of 3D Bioprinting Technology
}

\author{
Jaqueline L. Vieira ${ }^{1}$, Diego C. Carneiro ${ }^{1}$, Milena B. P. Soares ${ }^{1,2}$, Josiane D. V. Barbosa ${ }^{2 *}$ \\ ${ }^{1}$ Gonçalo Moniz Institute, Oswaldo Cruz Foundation (FIOCRUZ); ' Institute of Health Technology, SENAI CIMATEC, \\ Salvador, Bahia, Brazil
}

3D bioprinting consists in the printing of synthetic 3D structures used as biomaterials, along with cells, growth factors, and other components necessary to create a new functional organ. This technology can be applied to regenerative medicine and tissue engineering to treat diseases, test pharmaceuticals, and study the mechanisms underlying diseases. Currently, there are three basic types of 3D bioprinting technologies: laser, droplet, and extrusion. Laser-based bioprinters (LBP) use laser energy to induce the bioink transfer. Dropletbased bioprinters (DBP) expel the bioink dropwise throughout a nozzle. Inkjet-based bioprinters are the DBP commonly used for biological proposes, it is also a non-contact approach that releases controlled volumes of bioink drops in a continuous (CIJ) or under demand way (DOD). The extrusion-based bioprinters (EBB) also use pressure to force out the bioink, but consists of a syringe containing the material with a pneumatic or mechanical mechanism as dispensing system. Comparing to the other bioprinting technologies, extrusion printing is the most versatile and is indicated for bioprinting of scaffold prosthetic implants. The bioinks used in 3D bioprinting are composed of a solution with a biomaterial mixture, usually encapsulating cells. Biomaterials are essential components of 3D bioprinting technologies because they provide scaffolds as supporting physical structures for cells to attach, grow, differentiate, and develop into tissues. Numerous cell types have been used in 3D bioprinting to build cardiovascular, musculoskeletal, neural, hepatic, adipose and skin tissues. Bioprinting is an emerging technology that has the ability to revolutionize the way we address many health issues.

Keywords: Laser. Droplet. Extrusion. Bioprinters. Bioinks.

Abbreviations: CAD/CAM: computer-aided design or manufacturing; LBP: Laser-based bioprinter; DBP: droplet-based bioprinter; EBB: extrusion-based; CIJ: continuous inkjet; DOD: drop-on-demand; iPSC: induced pluripotent stem cell.

\section{Introduction}

The advance of the printing technology from $2 \mathrm{D}$ to $3 \mathrm{D}$ has been continuously changing research in many fields, including health sciences. The advent of 3D bioprinting brought a new universe of possibilities. 3D bioprinting consists in the printing of synthetic 3D structures used as biomaterials, along with cells, growth factors, and other components necessary, at least in theory, to create a new functional organ [1].

The first 3D printing was performed in 1986 by creating thin layers of materials in rheological

Received on 19 March 2021; revised 18 May 2021.

Address for correspondence: Josiane D. V. Barbosa. SENAI CIMATEC. Avenida Orlando Gomes, Número 1845 - Piatã, Zip Code: 41650-010, Salvador, Bahia, Brazil. Phone: +55 (71) 992517119. E-mail: josianedantas@fiieb.org.br.

J Bioeng. Tech. Appl. Health 2021;4(2):63-70.

(C) 2021 by SENAI CIMATEC. All rights reserved. conditions of appropriate viscosity that were solidified by a process of cure with ultraviolet light [2]. This former method was not yet appropriate for use in biological creations since it used solvents and reagents that did not grant cell viability. After some decades, the process was improved, and biomaterials were created and used in solvent-free processes, generating structures for biomedical applications, until bioprinting was created $[1,3]$.

$3 \mathrm{D}$ bioprinting has been used to fill the gaps in the current techniques, in which the models of interest have complex geometries, in order to achieve a faster and more scalable production [4]. This apparatus uses the so-called bioinks, components composed of biomaterials with certain properties, biochemical factors, and cells printed in a layer-by-layer process. They go through a final curing step, in which the structure turns solid with the support properties of interest $[1,4]$.

The first bioprinting methods were limited to scaffolds only with support properties. However, the development of the method, along with the 
increasing interest in tissue engineering and regenerative medicine, led to new models and proposals, aiming the creation of tissues and organs for drug screening, cancer models, regeneration of damaged tissue, organ and tissue transplantation, among other conditions [5]. Research advanced to improve the printing technique by building scaffolds with increasingly anatomical fitness and properties similar to the original tissues, enabling a certain biomimetic degree to the native tissues in heterocellular environments [6].

Those tissue engineering approaches can be performed in many ways, varying in printing method, matrix material, cell type, and other factors that can constitute the bioink, which come with advantages, disadvantages, and challenges. As summarized in Figure 1, in this review we aim to establish the 3D bioprinting technology.

Although bioprinting is generally performed by controlled depositions of layers of bioink, the principle used by the printing technology can vary. Currently, there are three basic types of bioink deposition: laser, droplet, and extrusion. These techniques vary among themselves, not only by the rinciple used to release the ink, but also in the resolution of the results obtained. Therefore, it is important to review their limitations before selecting the proper technology [4]. Regardless of the method used, they all need a computer model to be followed during printing, usually generated

Figure 1. Overview of the 3D bioprinting technology. The bioink includes cells and biomaterials under certain parameters and is used by the bioprinter technology to construct 3D scaffold structures by layerby-layer deposition, following a design made in the computer. After a curing step, the engineered organ or tissue have several useful biomedical applications.

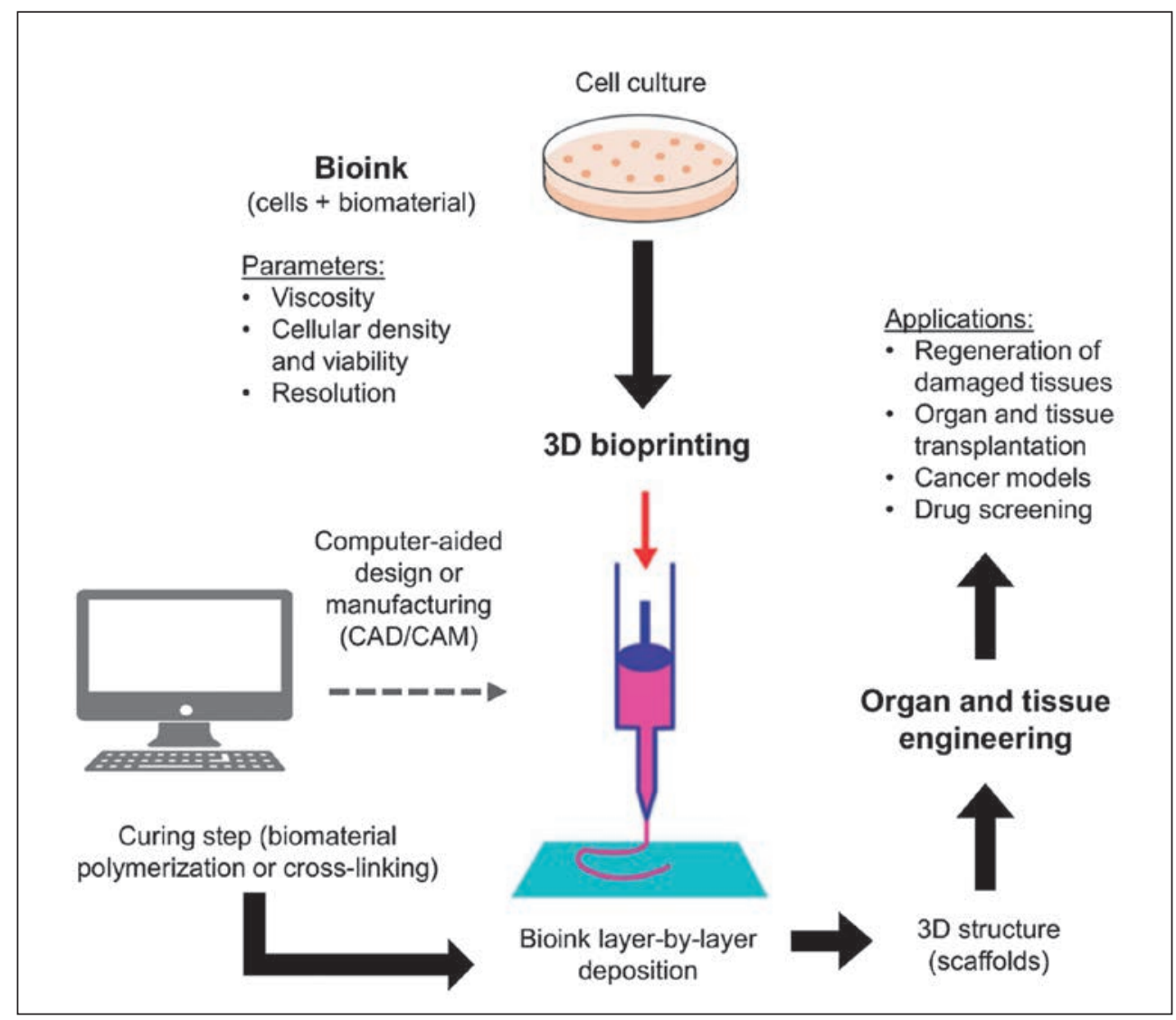


through tools such as computer-aided design or manufacturing (CAD/CAM) [7].

\section{Laser-based Bioprinters}

As the name says, the laser-based bioprinter (LBP) uses laser energy to induce the bioink transfer (Figure 2). It is the most unusual technology, but it has been gaining ground in tissue engineering [4]. The main components are a laser radiation highly focused with a monochromatic base, which can be pulsed or continuous, a laser transparent print ribbon carrying the bioink, and a collecting plate on a controlled movement platform [7]. The ribbon has a laser-absorbing layer that receives the irradiation, forming a high-pressure environment where bubbles of the bioink are generated and expelled to the collector [4].

Figure 2. Laser-based bioprinter primary construction. Demonstration of basic items and bioink deposition by the laser irradiation pressure.

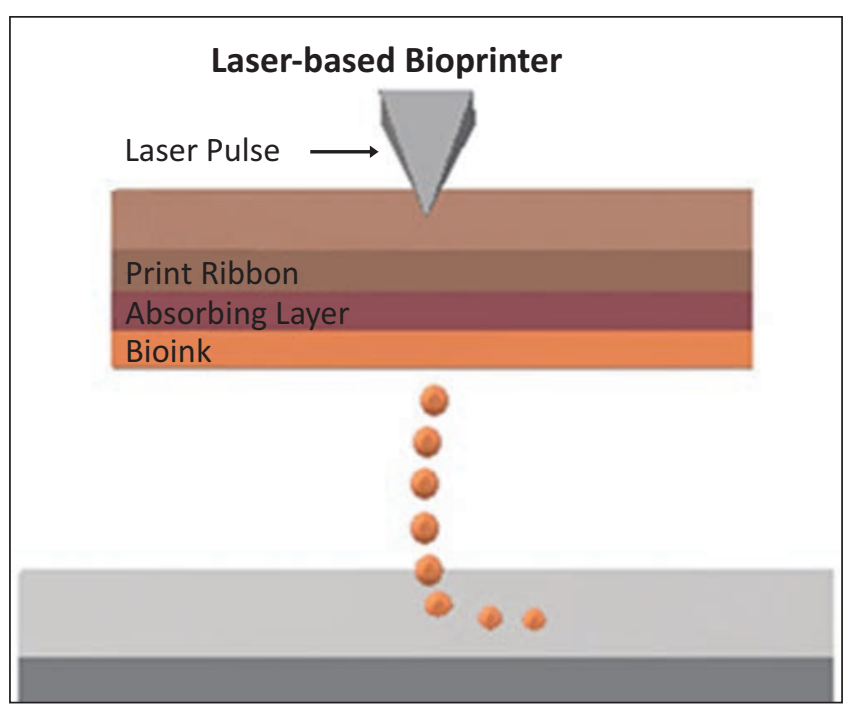

LBP makes a direct impression on the collecting surface, without the need for a needle to intermediate it, decreasing the impacts on the cells, since the passage of cells immersed in a substrate of a certain viscosity is one of the main causes of reduction in cell viability [8]. In addition to bypassing the problems resulting from shear stress, this contactless approach abolishes the chances of undue clotting, and allows the cells to be individually printed in droplets, increasing cell densities, and consequently increasing resolution [9].

Although the cells do not suffer damages by passing the needle, the interaction with the laser and the substrate can alter its integrity, reinforcing the importance of a suitable cell density to create a viable scaffold [10]. Cell density is one of the factors that alters the bionic viscosity, a parameter that is decisive for cell viability in printers that use needles. Since LBP does not use one, it can work with different ranges of viscosity, increasing the ability to carry more cells per mL [11].

The resolution of 3D bioprinting using LPB can be adjusted by several factors. Altering parameters such as surface tension, wettability of the substrate, bioink viscosity, among others, interfere with the resolution [9]. By adjusting these factors, the technique allows the creation of high-resolution models in micrometric rates with different cell type environments [12]. However, in order to mimic a complex model, the bioink needs to solidify rapidly in the desired shape, limiting the viscosity [4].

LBP has some disadvantages beyond the laser-cell interaction and high cost. Some LBP bioprinters use metal layers to absorb laser energy, generating nanoparticles in the process, which may be cytotoxic. To use multiple cell types, different ribbons must be prepared since cellular position is not so accurate, and scaling is still a problem [4]. The alternatives to overcome these issues are time-consuming, onerous, and still not practical; therefore, the technique is recommended for simpler and smaller scaffolds, even for diseases and drug studies [7].

\section{Droplet-based Bioprinters}

Droplet-based bioprinters (DBP) expel the bioink dropwise throughout a nozzle. It is possible to classify them according to the mechanism used to dispense the material. These printers can use pressure, thermal, piezoelectric, electrostatic, electrohydrodynamic, or even acoustic forces 
as motors. Regardless the method, they all have similar structures. They are composed of a nozzle submitted to the push where goes the bioink, and a collecting plate [7].

Inkjet-based bioprinters, among DBP, are the most used for regular 3D printing and bioprinting. It is also a non-contact approach that releases controlled volumes of bioink drops in a continuous (CIJ) or under demand way (DOD). The CIJ method uses a pressure instability to release the material, hampering the control of the printing process, and is unadvised for bioprinting [11].

The inkjet bioprinters can print scaffolds with different cell gradients, also allowing the input customization of biochemical factors in the scaffold structure, which better mimicks the cellular environment of interest, in addition to being a high-speed method $[13,14]$. Nonetheless, it is a versatile method that can be used in combination with more than one technique, such as electrospinning [15]

The drop-on-demand method only releases the material when requested. The trigger can be thermal, piezoelectric or electrostatic (Figure 3). Thermal DODs use the temperature rise to create different pressure points, forming a vapor blister that compells out the droplet when it bursts [16]. Piezoelectric crystals can be placed into the nozzle head, where they are submitted to a voltage pulse, causing a crystal contraction or dilation, resulting in a pressure difference inside the nozzle that releases the droplet onto the substrate. Electrostatic approaches use the change in the volume of the bioink container, created by the application of high voltage in the system, which is composed of electrodes and deflection plates to eject the ink [17].

Every motive force applied has its ups and downs that need to be taken into consideration when applied in DBP [16]. The thermal method is the most simple and cost-effective but creates uneven drops. Because this technique uses temperatures to create the perturbation, the cells are exposed to some adverse conditions. Even if the increase in temperature does not directly affect the cells, the shear stress does [17].

The piezoelectrical generates more uniform droplets released in a more controlled manner, without variation in temperature, allowing the use of a system with a wider nozzle and decreasing the risks of clotting and shear stress [4]. The major issue with this technique lies in the possible cell damage caused by the frequency used to print and in the necessary refinement of the matrix [18].

Electrostatic DOD has in its nozzle an electrode and a high voltage deflection plate in place of the piezoelectrical crystal. A voltage pulse flows

Figure 3. Types of DOD bioprinters. Components of the thermal, piezoelectric, and electrostatic DODs.

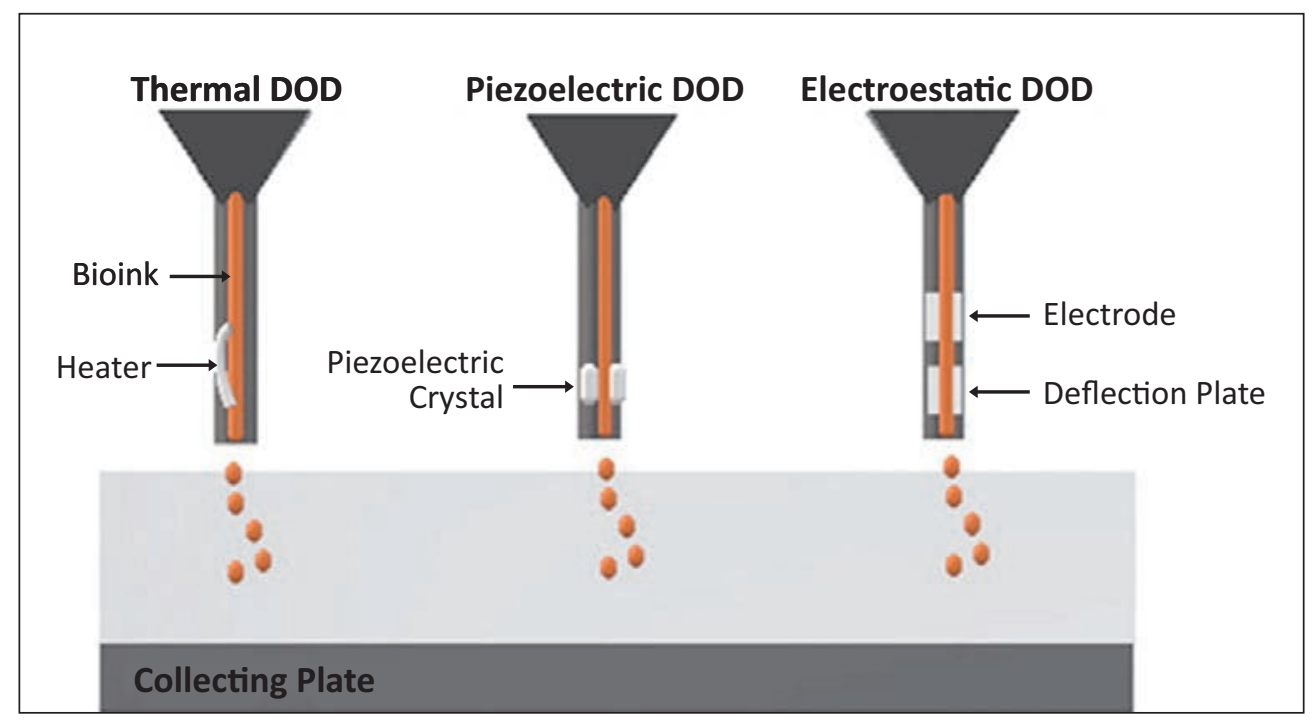


through the structure, altering the local pressure, dislocating the ink and causing the droplet deposition in the substrate [7]. Regardless of the principle, inkjet bioprinters function only for bioinks with limited viscosity, thus influencing the final cell concentration printed per $\mathrm{mL}$, to prevent clogging and reduce shear stress [19].

In bioinks with low viscosity, the use of some post-print gelation is a key point in which a widely used option is the cross-linkers. Although necessary, must be used carefully because some chemical changes can occur in the material in order to gelatinize the final scaffold, altering its structure and properties beyond possible cytotoxicity [4].

\section{Extrusion-based Bioprinters}

The extrusion-based bioprinters (EBB) are printers that also use pressure to force out the bioink, but in this case, the system consists of a syringe containing the material to be printed, which can use a pneumatic or mechanical mechanism as dispensing system (Figure 4) [12]. It is the most common bioprinter due to its ease to scale up the production, allowing the construction of larger scaffolds, and is available both in simpler models

Figure 4. Types of extrusion-based bioprinters. Mechanical or pneumatic dispensing systems use pressure to release the bioink.

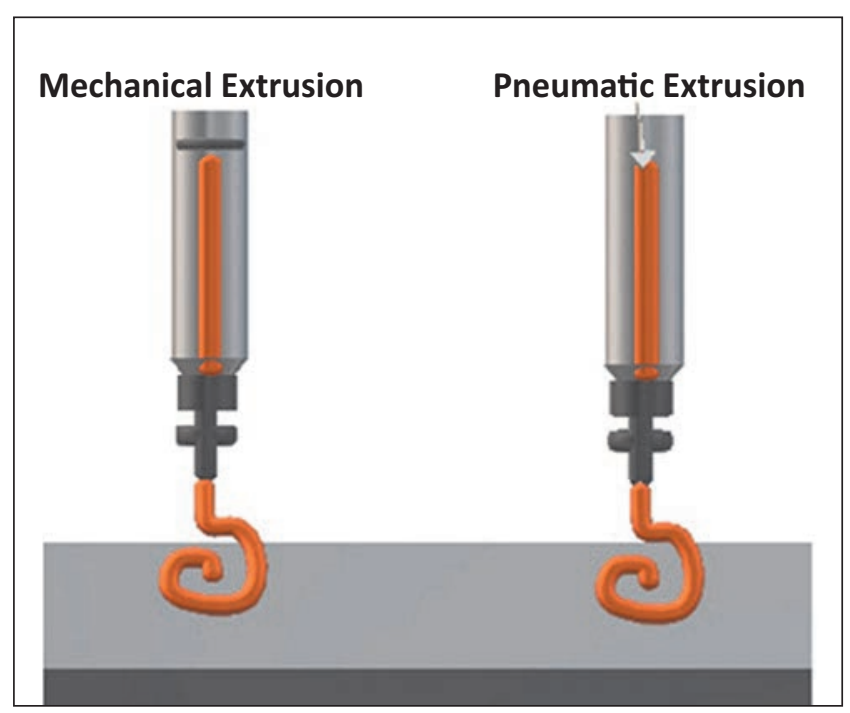

for research applications, or more robust models for industrial production $[7,20]$.

Beyond the multiple plastic syringe system for liquid output, the newest EBB bioprinters contain at least extruders for pellet deposition, to enable the use of a wider range of materials, a mobile collecting table, and photocuring systems. It is also possible to combine with other deposition techniques, such as electrospinning, in the same printer, favoring the synthesis of hybrid scaffolds [21].

Differently from the other methods, extrusion has a continuous deposition while in contact with the collector substrate. This approach makes it possible to control the temperature (both in the container and on the table), the pressure applied, the extrusion speed, and other parameters such as table type and position [12].

Mechanically controlled extrusion offers a better direct and spatial control of the dispensing system of the material; therefore, they are recommended for more viscous fluids [22]. Although the pneumatic approach has a delay between the gas compression and the material release, it could also be used to print viscous materials by adjusting the pressure and pressure time, which is limited only by the amount of compressed air endured by the system [23].

Thanks to the possibility to use more viscous fluids, extrusion bioprinters can use bioinks with higher cell density, generating tissues with greater similarity to the native tissue. Moreover, some studies have used EBB with more than one cell type printed simultaneously in the same scaffold, and biochemical compounds, including even DNA and RNA molecules [15, 24-27].

Extrusion bioprinting, however, has disadvantages. This technique does not withstand a bioink with high cell densities. The cell survival rate and the pressure that the cells are subjected to pass the syringe are lower than in the other methods and tends to decrease even more with increasing flow viscosity due to the magnification of the shear stress [28]. Another frequent issue to be address is the nozzle clogging, a consequence of this contact technique and its high viscous fluids, 
leading to misprinted scaffolds and equipment problems [29].

To bypass clogging and cell damage issues, it is possible to use a large nozzle, but it would result in a drop of resolution. In order to overcome the inferior resolution, some parameters must be adjusted, resulting again in a higher pressure and shear stress. Therefore, the optimization of printing parameters are crucial steps in this method $[5,7,29]$. Comparing to the other bioprinting technologies, extrusion printing is the most versatile and is indicated for bioprinting of scaffold prosthetic implants [12].

\section{Bioinks}

The bioinks used in 3D bioprinting are composed of a solution with a biomaterial mixture, usually encapsulating cells. During or immediately after bioprinting, the bioinks can be cross-linked or stabilized to create the tissue constructs. While synthetic or natural biomaterials can be combined to make hybrid scaffolds, aggregating cells can also be used as bioinks without addition of biomaterials. To achieve the correct functionalities of the target tissues, the bioinks must have proper mechanical, rheological, and biological properties [30].

Before, during, and after gelation, the following properties of the bioinks are essential for their printability: structural resolution, shape fidelity, and cell survival. The cross-linking extent and charge densities determine the swelling behavior of the biomaterials, which eventually influences the shape and size of the bioprinted tissue. Cell viability can be enhanced while maintaining printability by combining biomaterials optimized for cell survival with biomaterials that have mechanical stability and provides shape fidelity [31].

Biomaterials are essential components of 3D bioprinting technologies because they provide scaffolds as supporting physical structures for cells to attach, grow, differentiate, and develop into tissues [32]. Biocompatibility significantly limits the number of appropriate materials. They must suit both encapsulated cells and host's body without inducing inflammation or rejection. Since hydrogels have an elevated water content and low toxicity, they are common biomaterials used in tissue engineering to construct tissues that better mimic their extracellular matrix microenvironments [33].

The bioink parameters of viscosity, cell density, resolution, fabrication speed, and cell viability vary for each bioprinting technology. While DBP supports low viscosity and cell density but has a fast fabrication speed, LBP supports a higher viscosity but medium cell density and fabrication speed. EBP has the higher viscosity and cell density support but has the lowest fabrication speed. Moreover, EBP can achieve the highest resolution among all current bioprinters [31, 32].

Ranging from differentiated somatic cells to stem and progenitor cells, numerous cell types have been used in 3D bioprinting to build cardiovascular, musculoskeletal, neural, hepatic, adipose, and skin tissues [34]. However, bioinks that are both printable and able to convey the tissue architecture to restore an organ's function are scarce. In addition, vascularization of the bioprinted constructs with integration to the host's vasculature is a major challenge [35].

While embryonic stem cells are pluripotent and derived from the inner mass cells of the blastocyst stage of an embryo, mesenchymal stem cells are multipotent adult cells present in various tissues. Induced pluripotent stem cells (iPSCs) have promising applications in regenerative medicine. iPSCs are somatic cells genetically reprogrammed back to a stem cell phenotype [36]. They represent an unlimited source of patient-specific cells that do not trigger host rejection, and they can be applied in disease modeling with phenotype variability. The 3D bioprinting technologies can employ stem cells, which have the ability to progress into different cell lineages, progenitor cells (with limited capacity to follow other cell lines), or somatic cells that have already achieved their last differentiation stage [34].

Shear stress during the printing process is detrimental to stem cells, especially to iPSCs, and 
it can even affect their gene expression profile. Therefore, the bioprinting procedures need to be tested beforehand in order to effectively produce tissues and organoids for drug testing and disease models as well as for the regeneration of damaged tissues and treatment of diseases [35].

\section{Conclusions}

Bioprinting is an emerging technology that has the ability to revolutionize the way we address many health issues. It allows the opening of a new door to regenerative medicine, where the main goal is not simply to replace non-functional or damaged tissues by temporary implants with side effects. Bioprinting is about creating a system in which the body can rebuild itself properly, producing new healthy, functional, and longlasting tissues [37]. In addition to the applications in regenerative medicine, bioprinting has also been used in the research of drug mechanisms and disease models, pursuing to validate more humanlike models instead of the animal models currently used [12].

Although bioprinting has many advantages, there are some drawbacks that need to be overcome. The main one involves the bioink preparation and deposition. However, there is also the necessity to find proper materials and cell combinations that generate suitable bioinks with viscosity ranges that allow a printing process with less cellular damages and with a final scaffold able to maintain the structure's properties without collisions. Because it is a new technology, it has yet to be improved to increase post-print scaffold viability and functionality and, therefore, it is suitable for clinical uses. Finally, long-term tests, in all steps, need to be done wherefore new advances can be achieved with this promising technique $[7,12]$.

\section{Acknowledgements}

The authors would like to thank to Coordination for the Improvement of Higher Education Personnel (CAPES) for the support.

\section{References}

1. Nakamura M, Iwanaga S, Henmi C, Arai $\mathrm{K}$, Nishiyama Y. Biomatrices and biomaterials for future developments of bioprinting and biofabrication. Biofabrication. 2010;2(1):014110. doi:10.1088/17585082/2/1/014110.

2. Hull CW. Apparatus for production of three-dimensional objects by stereolithography. 1986. US4575330A.

3. Kruth JP. Material Incress Manufacturing by Rapid Prototyping Techniques. CIRP Ann. Manuf. Technol. 1991;40(2):603-614.

4. Murphy SV, Atala A. 3D bioprinting of tissues and organs. Nat Biotechnol. 2014;32(8):773-785. doi:10.1038/nbt.2958

5. Matai I, Kaur G, Seyedsalehi A, McClinton A, Laurencin CT. Progress in 3D bioprinting technology for tissue/organ regenerative engineering. Biomaterials. 2020;226:119536. doi:10.1016/j. biomaterials.2019.119536

6. Ozbolat IT, Peng W, Ozbolat V. Application areas of 3D bioprinting. Drug Discov Today. 2016;21(8):12571271. doi:10.1016/j.drudis.2016.04.006

7. Vijayavenkataraman S, Yan WC, Lu WF, Wang $\mathrm{CH}$, Fuh JYH. 3D bioprinting of tissues and organs for regenerative medicine. Adv Drug Deliv Rev. 2018;132:296-332. doi:10.1016/j.addr.2018.07.004

8. Sun W, Starly B, Daly AC, et al. The bioprinting roadmap. Biofabrication. 2020;12(2):022002. Published 2020 Feb 6. doi:10.1088/1758-5090/ab5158

9. Hopp B, Smausz T, Kresz N, et al. Survival and proliferative ability of various living cell types after laser-induced forward transfer. Tissue Eng. 2005;11(1112):1817-1823. doi:10.1089/ten.2005.11.1817

10. Duocastella M, Colina M, Fernández-Pradas JM, Serra P, Morenza JL. Study of the laser-induced forward transfer of liquids for laser bioprinting. Appl. Surf. Sci. 2007;253(19):7855-7859.

11. Guillemot F, Souquet A, Catros S, Guillotin B. Laserassisted cell printing: principle, physical parameters versus cell fate and perspectives in tissue engineering [published correction appears in Nanomedicine (Lond). 2010 Jul;5(5):831-2]. Nanomedicine (Lond). 2010;5(3):507-515. doi:10.2217/nnm.10.14

12. Matai I, Kaur G, Seyedsalehi A, McClinton A, Laurencin CT. Progress in 3D bioprinting technology for tissue/organ regenerative engineering. Biomaterials. 2020;226:119536. doi:10.1016/j. biomaterials.2019.119536

13. Phillippi JA, Miller E, Weiss L, Huard J, Waggoner A, Campbell P. Microenvironments engineered by inkjet bioprinting spatially direct adult stem cells toward muscle- and bone-like subpopulations. Stem Cells. 2008;26(1):127-134. doi:10.1634/stemcells.2007-0520 
14. Campbell PG, Miller ED, Fisher GW, Walker LM, Weiss LE. Engineered spatial patterns of FGF2 immobilized on fibrin direct cell organization. Biomaterials. 2005;26(33):6762-6770. doi:10.1016/j. biomaterials.2005.04.032

15. $\mathrm{Xu} \mathrm{T}$, Binder $\mathrm{KW}$, Albanna MZ, et al. Hybrid printing of mechanically and biologically improved constructs for cartilage tissue engineering applications. Biofabrication. 2013;5(1):015001. doi:10.1088/17585082/5/1/015001

16. Derby B. Inkjet printing of functional and structural materials: Fluid property requirements, feature stability, and resolution. Annu. Rev. Mater. Res. 2010;40:395-414.

17. Matsusaki M, Sakaue K, Kadowaki K, Akashi M. Threedimensional human tissue chips fabricated by rapid and automatic inkjet cell printing. Adv Healthc Mater. 2013;2(4):534-539. doi:10.1002/adhm.201200299

18. $\mathrm{Xu} \mathrm{T}$, Jin J, Gregory C, Hickman JJ, Boland T. Inkjet printing of viable mammalian cells. Biomaterials. 2005;26(1):93-99. doi:10.1016/j. biomaterials.2004.04.011

19. Pedde RD, Mirani B, Navaei A, et al. Emerging Biofabrication Strategies for Engineering Complex Tissue Constructs. Adv Mater. 2017;29(19):10.1002/ adma.201606061. doi:10.1002/adma.201606061

20. Murphy SV, Atala A. 3D bioprinting of tissues and organs. Nat Biotechnol. 2014;32(8):773-785. doi:10.1038/nbt.2958

21. OctopusTM - 3D Biotechnology Solutions. [Accessed: 06-Jun-2021]. Available at: http:// www.3dbiotechnologiessolutions.com/bioimpressao3d/octopus/

22. Mironov V. Printing technology to produce living tissue. Expert Opin Biol Ther. 2003;3(5):701-704. doi:10.1517/14712598.3.5.701

23. Chang CC, Boland ED, Williams SK, Hoying JB. Direct-write bioprinting three-dimensional biohybrid systems for future regenerative therapies. J Biomed Mater Res B Appl Biomater. 2011;98(1):160-170. doi:10.1002/jbm.b.31831

24. Maurmann $\mathrm{N}$ et al. Mesenchymal stem cells cultivated on scaffolds formed by 3D printed PCL matrices, coated with PLGA electrospun nanofibers for use in tissue engineering. Biomed. Phys. Eng. Express. 2017;3(4):045005.

25. Zhang X, Liu Y, Luo C, et al. Crosslinker-free silk/ decellularized extracellular matrix porous bioink for 3D bioprinting-based cartilage tissue engineering. Mater Sci Eng C Mater Biol Appl. 2021;118:111388. doi:10.1016/j.msec.2020.111388
26. Daly AC, Critchley SE, Rencsok EM, Kelly DJ. A comparison of different bioinks for 3D bioprinting of fibrocartilage and hyaline cartilage. Biofabrication. 2016;8(4):045002. Published 2016 Oct 7. doi:10.1088/1758-5090/8/4/045002

27. Zhang J, Zhao S, Zhu M, et al. 3D-printed magnetic $\mathrm{Fe} 3 \mathrm{O} 4 / \mathrm{MBG} / \mathrm{PCL}$ composite scaffolds with multifunctionality of bone regeneration, local anticancer drug delivery and hyperthermia. J Mater Chem B. 2014;2(43):7583-7595. doi:10.1039/c4tb01063a

28. Smith CM, Stone AL, Parkhill RL, et al. Threedimensional bioassembly tool for generating viable tissue-engineered constructs. Tissue Eng. 2004;10(910):1566-1576. doi:10.1089/ten.2004.10.1566

29. Chang R, Nam J, Sun W. Effects of dispensing pressure and nozzle diameter on cell survival from solid freeform fabrication-based direct cell writing. Tissue Eng Part A. 2008;14(1):41-48. doi:10.1089/ten.a.2007.0004

30. Gungor-Ozkerim PS , Inci I , Zhang YS , Khademhosseini A, Dokmeci MR . Bioinks for 3D bioprinting: an overview. Biomater Sci. 2018;6(5):915946. doi:10.1039/c7bm00765e

31. Hölzl K, Lin S, Tytgat L, Van Vlierberghe S, Gu L, Ovsianikov A. Bioink properties before, during and after 3D bioprinting. Biofabrication. 2016;8(3):032002. Published 2016 Sep 23. doi:10.1088/17585090/8/3/032002 XXXX

32. Yilmaz B, Tahmasebifar A, Baran ET. Bioprinting Technologies in Tissue Engineering. Adv Biochem Eng Biotechnol. 2020;171:279-319. doi:10.1007/10 2019108

33. Li J, Chen M, Fan $\bar{X}$, Zhou H. Recent advances in bioprinting techniques: approaches, applications and future prospects. J Transl Med. 2016;14:271. Published 2016 Sep 20. doi:10.1186/s12967-016-1028-0

34. Ong CS, Yesantharao P, Huang CY, et al. 3D bioprinting using stem cells. Pediatr Res. 2018;83(1-2):223-231. doi:10.1038/pr.2017.252

35. Dey M, Ozbolat IT. 3D bioprinting of cells, tissues and organs. Sci Rep. 2020;10(1):14023. Published 2020 Aug 18. doi:10.1038/s41598-020-70086-y

36. Ullah I, Subbarao RB, Rho GJ. Human mesenchymal stem cells - current trends and future prospective. Biosci Rep. 2015;35(2):e00191. Published 2015 Apr 28. doi:10.1042/BSR20150025

37. Balakhovsky YM, Ostrovskiy AY, Khesuani YD. Emerging Business Models Toward Commercialization of Bioprinting Technology. 3D Printing and Biofabrication. 2017:1-22 